\title{
Psicooncología
}

ISSN: 1696-7240

\section{Necesidad de apoyo psicológico y calidad de vida en el cuidador primario de pacientes pediátricos con cáncer}

\author{
Ester Alejandra Rosado Pulido ${ }^{1 *}$; Cointa Arroyo Jiménez ${ }^{2}$; Arturo Sahagún Morales ${ }^{3}$; Argelia \\ Lara Puente ${ }^{4}$; Sofia Campos Ugalde ${ }^{5}$; Rosalba Ochoa Palacios ${ }^{6}$; Juan José Sánchez Sosa
}

Recibido: 30 de noviembre de 2020 / Aceptado: 1 de marzo de 2021

Resumen: El cáncer infantil es un problema de salud pública en México. Esta enfermedad es motivo de angustia y problemas psicosociales tanto en el paciente como en su cuidador primario. Si las necesidades del cuidador no son identificadas y atendidas se impacta sobre el bienestar y la salud del infante. EI objetivo de la presente investigación fue evaluar las necesidades de apoyo (NA) y calidad de vida del cuidador primario informal (CPI) de pacientes pediátricos con cáncer. Método: El diseño fue no experimental y correlacional. Participaron 100 CPIs de pacientes pediátricos con cáncer hospitalizados, la mayoría fueron mujeres y madres de los pacientes. Resultados: Se encontró que las NA mayormente insatisfechas fueron la necesidad de resolver las preocupaciones por el futuro, seguido por las necesidades emocionales y las necesidades de información. Aquellas percibidas con una menor urgencia por resolver fueron las necesidades relacionadas con el acceso médico de los pacientes. El funcionamiento emocional, físico y social fueron las áreas más afectadas en la calidad de vida (CV). Con respecto al impacto familiar, las relaciones familiares mostraron ser uno de los dominios menos afectados. El análisis de regresión múltiple por método intro demostró que las necesidades emocionales y de información no satisfechas, las preocupaciones, actividades diarias, y relaciones familiares de los CPIs explican el $74 \%$ de la varianza en calidad de vida. Conclusión: Se recomienda integrar a su protocolo clínico de rutina la detección de necesidades, evaluación de $\mathrm{CV}$, así como la implementación de intervenciones psicoeducativas y, en su caso, la atención psicológica personalizada.

Palabras clave: Niños hospitalizados con cáncer, cuidadores primarios, necesidades de apoyo, calidad de vida, apoyo psicológico.

1 Ester Alejandra Rosado Pulido. Universidad Nacional Autónoma de México.

E-mail: ester_rosadop@hotmail.com

2 Cointa Arroyo Jiménez. Instituto Nacional de Pediatría. Ciudad de México.

E-mail: cointarroyo@gmail.com

3 Arturo Sahagún Morales. Universidad Nacional Autónoma de México.

E-mail: asm.psico@gmail.com

4 Argelia Lara Puente. Instituto Nacional de Pediatría. Ciudad de México.

E-mail: argelara@gmail.com

5 Sofia Campos Ugalde. Instituto Nacional de Pediatría. Ciudad de México.

E-mail: psicsofiacampos@gmail.com

6 Rosalba Ochoa Palacios. Instituto Nacional de Pediatría. Ciudad de México. E-mail: ochoarossgj@gmail.com

7 Juan José Sánchez Sosa. Universidad Nacional Autónoma de México.

E-mail: jujosaso@gmail.com

* Dirección de correspondencia: Ester Alejandra Rosado Pulido. C/ Millet 9B, Colonia Extremadura Insugentes, Ciudad de México, México. C.P.: 03740. E-mail: ester_rosadop@hotmail.com 


\title{
[en] Need of psychological support and assessment of quality of life of family caregivers of children with cancer
}

\begin{abstract}
Children's cancer is a public health problem in Mexico. This disease is also cause of distress and psychosocial problems for the patient, as well as their primary caregiver. The family is the first circle of support for a patient, therefore, if their needs are unmet, the infant's health and wellbeing will suffer in turn. The purpose of this investigation project was to evaluate supportive needs (SN) and quality of life (QOL) of family caregivers (FCs) of pediatric cancer patients. Method: This investigation's design was correlational and non-experimental. The study's participants were $100 \mathrm{FCs}$ for pediatric cancer patients currently hospitalized at the National Institute of Pediatrics in Mexico City, the majority were women and mothers of the patients. Results: The results showed that facing the worries about the future, emotional needs, as well as information needs were the most unsatisfied SN's. The one with least urgency to solve was the patients access and medical continuity needs. Proper emotional, physical and social function were the most affected areas of their quality of life. On the other hand, the family relationship of the family impact domain was one of the less affected. Furthermore, intro multiple regression showed that unmet needs relating to information and emotions, in addition to worries, daily activities and family relationships explained $74 \%$ of the variance in QOL. Conclusion: It is advised that detection of needs, evaluation of quality of life, psychological support and the implementation of psychoeducational interventions be integrated into routine clinical attention in this population.
\end{abstract}

Keywords: Hospitalized children with cancer, family caregivers, unmet needs, quality of life, psychological support.

Sumario: 1. Introducción 2. Método 3. Resultados 4. Discusión y conclusiones 5. Referencias bibliográficas

Como citar: Rosado Pulido EA, Arroyo Jiménez C, Sahagún Morales A, Lara Puente A, Campos Ugalde S, Ochoa Palacios R, Sánchez Sosa JJ. Necesidad de apoyo psicológico y calidad de vida en el cuidador primario de pacientes pediátricos con cáncer. Psicooncología 2021; 18: 137-156. doi: $/ 10.5209 /$ psic. 74536

\section{Introducción}

El cáncer infantil y su tratamiento genera afectaciones emocionales y físicas no solo en los pacientes, sino también en los Cuidadores Primarios Informales (CPIs), quienes la mayoría de las veces son las madres ${ }^{(1-5)}$.

En 1999 la Organización Mundial de la Salud (OMS) conceptualizó al cuidador primario como "la persona del entorno de un paciente que asume voluntariamente el papel de responsable del mismo, en un amplio sentido; este individuo está dispuesto a tomar decisiones por el paciente y a cubrir necesidades básicas del mismo, ya sea de manera directa o indirecta" ${ }^{(6)}$. Se define como "informales" a aquellos cuidadores que no disponen de capacitación específica, les motiva el componente afectivo y ofrecen atención sin límite de horario, tienen un elevado grado de compromiso hacia la tarea y no son remunerados por ejercerla ${ }^{(7)}$.

El CPI participa directa y activamente en la atención del enfermo, cumpliendo diferentes tareas para su función. Se ha encontrado que como parte de su rol realiza diversas actividades como: acompañar al paciente, asearlo, vestirlo, alimentarlo, cargarlo, transportarlo, llevarlo al médico, comprar y brindar medicamentos, así como programar las citas médicas ${ }^{(8)}$. 
El impacto del diagnóstico, los costos financieros, las demandas del tratamiento intensivo y prolongado, así como el pronóstico temeroso e incierto implican desafíos para las familias. Estos factores derivan en una experiencia de aprensión y preocupación por el futuro del paciente infantil ${ }^{(9)}$, al igual que un impacto negativo en la calidad de vida $(\mathrm{CV})$ de los cuidadores ${ }^{(10)}$.

Se ha observado que la calidad de vida de los cuidadores se ve maximizada cuando se satisfacen sus necesidades de apoyo en el contexto de atención del cáncer. Por lo tanto, determinar qué necesidades no están siendo cubiertas debería ser el paso inicial en el diseño de programas para mejorar la calidad de vida de los cuidadores ${ }^{(11)}$.

Las Necesidades de Apoyo (NA) del cuidador se han definido como las necesidades relacionadas con el estado de salud y de atención médica que no se cumplen ${ }^{(12,13)}$. Sloper ${ }^{(14)}$, realizó un estudio en el cual encontró que el $48 \%$ de los cuidadores de pacientes pediátricos con cáncer sentían no cubrir las NA que experimentaban situándose en primer lugar la necesidad de asesoramiento y apoyo emocional para ellos mismos. Otras investigaciones observaron que los CPIs han manifestado una fuerte necesidad de expresar con los profesionales de salud el miedo a la incertidumbre de futuras recaídas, segundos tumores y posible muerte de su hijo ${ }^{(15,16)}$.

Algunos autores han descrito las diferentes reacciones de los padres durante el proceso de enfermedad, argumentando que generalmente ocurren en fases de acuerdo al tiempo que los pacientes pediátricos llevan de diagnóstico, pasando por sentimientos de conmoción y negación a sentimientos de culpa, tristeza y, finalmente, expresiones de resolución y reorganización ${ }^{(17,18)}$.

Por otro lado, un metaanálisis realizado por Pai et al. ${ }^{(19)}$ reportó que, de acuerdo con los estudios revisados, el tiempo transcurrido desde el diagnóstico influía claramente en el funcionamiento psicológico y familiar de los cuidadores de niños con cáncer, especialmente dentro del primer año después del diagnóstico.

Resulta importante valorar correctamente aspectos socioculturales y psicológicos, así como realizar una buena detección de las necesidades de una población poco estudiada como es la de los cuidadores, en especial en ambientes pediátricos donde la atención se centra en los pacientes dejando de lado la influencia y repercusiones que la familia tiene en los niños con cáncer ${ }^{(20)}$.

Se observó con base en la búsqueda de la literatura científica realizada en las bases de datos: MEDLINE (PubMed) y PsycInfo (OVID) una evidente falta de estudios que aborden en conjunto las variables de NA y calidad de vida en CPIs de niños con cáncer; de acuerdo a nuestra búsqueda, la gran mayoría de estudios que abordan estas variables han sido con cuidadores de pacientes de adultos con cáncer. Por otro lado, el cáncer infantil es un problema de salud pública en México, ya que es la primera causa de muerte por enfermedad en este grupo de edad. No obstante, en la búsqueda realizada no se encontraron estudios que consideren la variable de necesidades de apoyo insatisfechas en el cuidador a pesar de la creciente prevalencia de pacientes pediátricos con cáncer ${ }^{(21)}$. El objetivo del estudio fue identificar la relación entre las necesidades de apoyo y calidad de vida del CPI de pacientes pediátricos con cáncer que reciben atención médica en un hospital de tercer nivel en la Ciudad de México. Los objetivos específicos fueron los siguientes:

1) Identificar las necesidades de apoyo y calidad de vida del CPI.

2) Describir la relación entre las NA, la calidad de vida del CPI y el tiempo desde el diagnóstico del paciente infantil.

3) Identificar los factores que predicen la calidad de vida del CPI. 


\section{Método}

\section{Diseño}

Estudio no experimental, transversal y correlacional.

\section{Participantes}

Se evaluaron a 100 cuidadores primarios informales de pacientes pediátricos con cáncer que recibían atención médica en el Instituto Nacional de Pediatría en la Ciudad de México, 94\% fueron mujeres y $6 \%$ hombres. Estos fueron elegidos de manera no probabilística por conveniencia. La edad mínima registrada fue de 18 años y la máxima de $59(\bar{x}=34 ; 67, \mathrm{DT}=8,05)$. La mayoría de los CPIs eran las madres de los pacientes $(91 \%)$, estaban casadas $(43 \%)$ o en unión libre $(31 \%)$, eran amas de casa (55\%) o se encontraban desempleadas (32\%), y tenían una educación básica de nivel secundaria (28\%) o de nivel media superior (29\%). La proporción respecto al sexo de los pacientes infantiles que cuidaban fue de fue de 53 varones y 47 niñas, el mínimo de edad fue de 1 año y el máximo de 18 años. En relación con el diagnóstico el $48 \%$ tenía leucemia, 13\% linfomas, 10\% tumores óseos, $5 \%$ tumores cerebrales y el $24 \%$ otro tipo de cáncer. Véase la Tabla 1.

Tabla 1. Datos Sociodemográficos de los CPIs de pacientes pediátricos con cáncer $(\mathrm{N}=100)$.

\begin{tabular}{lc}
\hline \multicolumn{1}{c}{ Datos sociodemográficos } & Frecuencia \\
\hline Parentesco & 91 \\
Madre & 6 \\
Padre & 2 \\
Abuela & 1 \\
Hermana & \\
Estado civil & 43 \\
Casada/o & 31 \\
Unión libre & 24 \\
Soltera/o & 2 \\
Viuda/o & \\
Ocupación & 55 \\
Amas de casa & 13 \\
Empleada/o & 32 \\
Desempleada/o & \\
Padecimiento de alguna enfermedad & 88 \\
No & 12 \\
Sí
\end{tabular}




\begin{tabular}{lc}
\hline \multicolumn{1}{c}{ Datos sociodemográficos } & Frecuencia \\
\hline Escolaridad & 1 \\
Nula & 15 \\
Primaria & 5 \\
Primaria incompleta & 28 \\
Secundaria & 3 \\
Secundaria incompleta & 29 \\
Bachillerato & 9 \\
Bachillerato incompleto & 8 \\
Universidad & 2 \\
Universidad incompleta & \\
Lugar de residencia & 66 \\
Ciudad de México y Estado de México & 34 \\
Otros estados & \\
Atención Psicológica & 68 \\
Ninguna & 20 \\
Asesoramiento & 10 \\
Apoyo psicológico & 1 \\
Apoyo psiquiátrico & 1 \\
Intervención en Crisis & \\
\hline
\end{tabular}

\section{Instrumentos}

1. Cuestionario de Datos Sociodemográficos. El cual consta de 18 preguntas de las cuales 10 son relacionadas al cuidador; edad, sexo, estado civil, escolaridad, empleo, residencia, padecimiento de enfermedad, parentesco con el paciente, apoyo psicológico y tiempo brindado al paciente. Las 8 preguntas restantes estaban dirigidas a información relacionada al paciente infantil como su edad, sexo, hermanos, tipo de cáncer diagnosticado, tiempo transcurrido desde el diagnóstico, tratamiento, número de recaídas y percepción de la enfermedad del paciente.

2. Encuesta de Necesidades de Apoyo Insatisfechas de la Persona de Apoyo, versión corta (SPUNS). Creada por Campbell et al. ${ }^{(22)}$ y validada en México en cuidadores primarios informales de pacientes con cáncer ${ }^{(21)}$. El instrumento cuenta con 23 ítems y con seis factores que representan el $65 \%$ de la varianza total. Los factores son: 1) Información, 2) Preocupaciones sobre el futuro, 3) Trabajo y Finanzas, 4) Acceso de la atención médica, 5) Necesidades Personales, y 6) Necesidades Emocionales. Los valores alfa de Cronbach son de 0,70 y 0,88 entre los factores. Demuestra una buena validez discriminativa, validez de contenido y validez de constructo.

3.Peds $Q L^{T M}$ 2.0, Módulo de Impacto familiar desarrollado por Varni et al.(23,24). El instrumento traducido busca ser entendido manteniendo un nivel de lectura y comprensión accesible para los encuestados, incluso de un nivel de educación básica. El algoritmo de proceso de validación lingüística constó de tres fases: 1) traducción directa, 2) traducción hacia atrás y 3 ) prueba con pacientes. La validación fue realizada 
por dos traductores profesionales, un hablante nativo de origen mexicano, y un bilingüe del idioma origen ${ }^{(24)}$. Este es un instrumento de autoaplicación constituido por 36 ítems divididos en 6 dominios que miden la funcionalidad de los padres: 1) Funcionamiento Físico, 2) Funcionamiento Emocional, 3) Funcionamiento Social, 4) Funcionamiento Cognitivo, 5) Comunicación, 6) Preocupaciones, y 2 dominios que miden la funcionalidad familiar reportada por los progenitores; 7) Actividades Diarias, y 8) Relaciones familiares. El instrumento fue diseñado con base en un modelo multidimensional y modular que incluye las dimensiones sugeridas por la Organización Mundial de la Salud para la Evaluación de la Calidad de Vida ${ }^{(25)}$. Existen tres medidas que se pueden obtener del instrumento: A) El Puntaje total del Impacto, el cual se obtiene sumando los 36 ítems del test, B) El Puntaje del Impacto en la Calidad de Vida relacionado con la Salud (HRQOL), el cual incluye los 20 ítems de los primeros 4 dominios y C) El Puntaje en el área de Repercusiones Familiares, en el cual se suman los 8 ítems de las dos últimas escalas ${ }^{(23)}$. Se demostró la consistencia interna para la Puntuación Total de la Escala $(\alpha=0,97)$, el resumen de Puntuación del Impacto de la Calidad de Vida relacionado con la Salud $(\alpha=0,96)$ y el resumen de Puntuación del funcionamiento familiar $(\alpha=0,90)$. Los valores alfa de Cronbach entre los factores fueron de entre 0,82 a 0,97. Además, el instrumento demostró una buena validez de constructo.

\section{Adaptación psicométrica}

Se estudiaron las propiedades psicométricas de la SPUNS y el PEDSQL para asegurar un uso válido y confiable de ambos instrumentos en la población objetivo. En ambos instrumentos se evaluó: 1) validez referente a la estructura interna mediante el Análisis Factorial Confirmatorio (AFC) con estimación de Mínimos Cuadrados Ponderados Robusto (WLSMV) y con matrices de correlaciones policóricas ${ }^{(26,27)}$, El ajuste de los modelos se evaluó con los indicadores RMSR y RMSEA $\leq 0,08$ (buen ajuste), CFI y TLI $\geq 0,95$ (buen ajuste), y $\chi^{2} / \mathrm{gl} \leq 3$ (buen ajuste) ${ }^{(26)} ; 2$ ) validez convergente mediante la Varianza Extraída Promedio (AVE por siglas en inglés) siguiendo el criterio de $\mathrm{AVE} \geq 0,50$ para afirmar convergencia de los ítems ${ }^{(28)}$; 3 ) validez discriminante mediante la comparación de la AVE y las correlaciones al cuadrado $\left(\mathrm{r}^{2}\right)$ entre factores afirmando discriminación al ser las AVE's mayores a las $\mathrm{r}^{2}{ }^{(29)}$; y 4) consistencia interna, total y por subescala, con los coeficientes Alfa de Cronbach Ordinal $(\alpha)$ y Omega de McDonald $(\omega)^{(26,30)}$ considerando que valores de consistencia $\geq 0,9$ son Excelentes, $\geq 0,8$ son Buenos, $\geq 0,7$ son Aceptables, $\geq 0,6$ son Cuestionables, $\geq 0,5$ son Pobres y $<0,5$ son inaceptables. Véanse las Tablas 2 y 3 .

Se observó que ambas escalas psicométricas cumplen todos los criterios de ajuste de modelos. No obstante, en la SPUNS se eliminaron los factores Trabajo y Finanzas y Necesidades Personales, por falta de ajuste. Por su parte, los ocho factores del PEDSQL ajustaron sin hacer modificaciones.

En ambas escalas todas las AVE's cumplieron satisfactoriamente el criterio de $\geq$ 0,50. Únicamente las ${ }^{2}$ 's entre los factores 1-3, 2-3 y 3-4 de la SPUNS no cumplen con el criterio de validez discriminante, lo que sugiere poca validez discriminante del factor 3, no obstante, debido al ajuste del modelo, la validez convergente, la coherencia semántica de los ítems con el factor y la consistencia interna, es posible trabajar con el factor 3 manteniendo altas reservas en la interpretación de sus puntajes. 


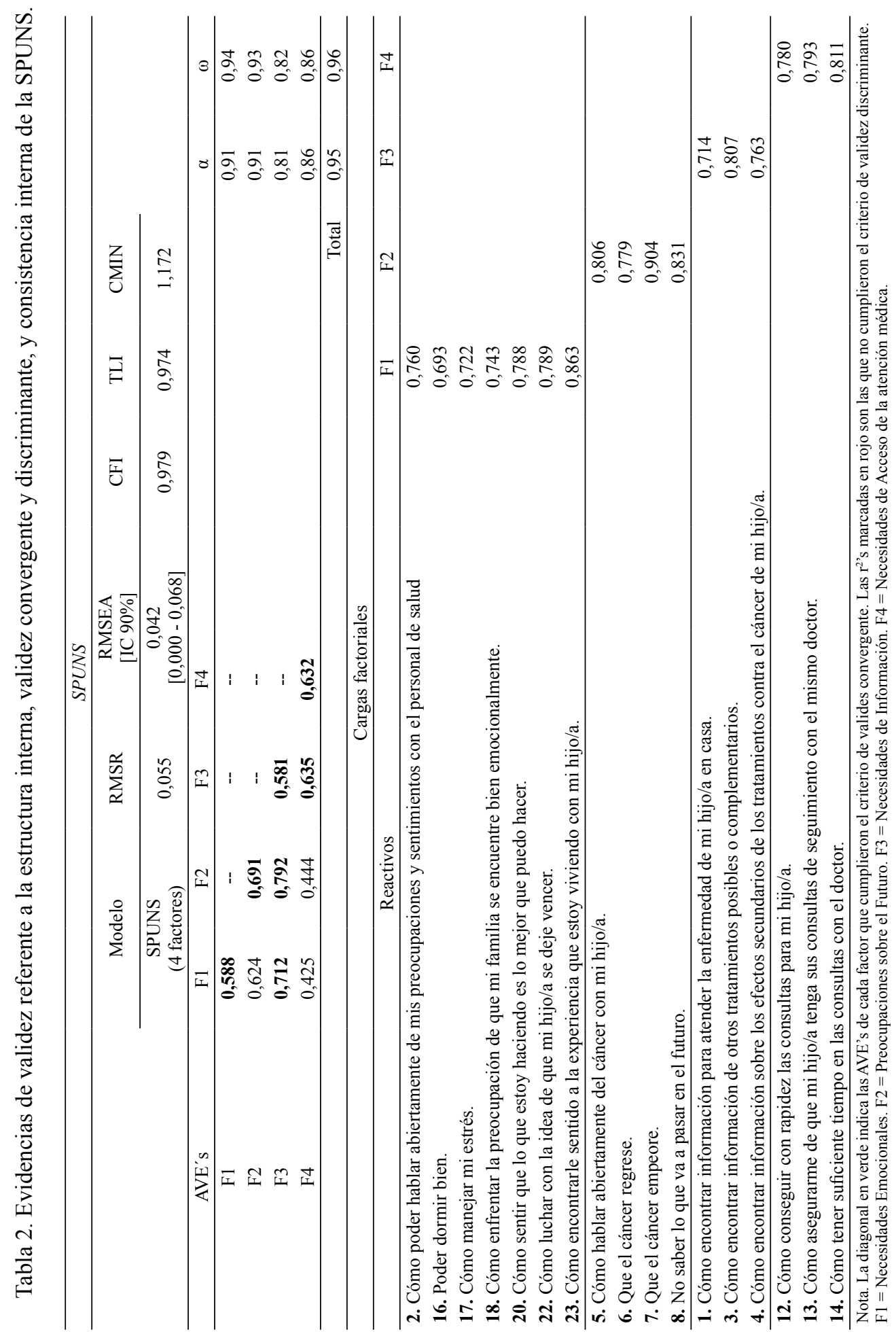




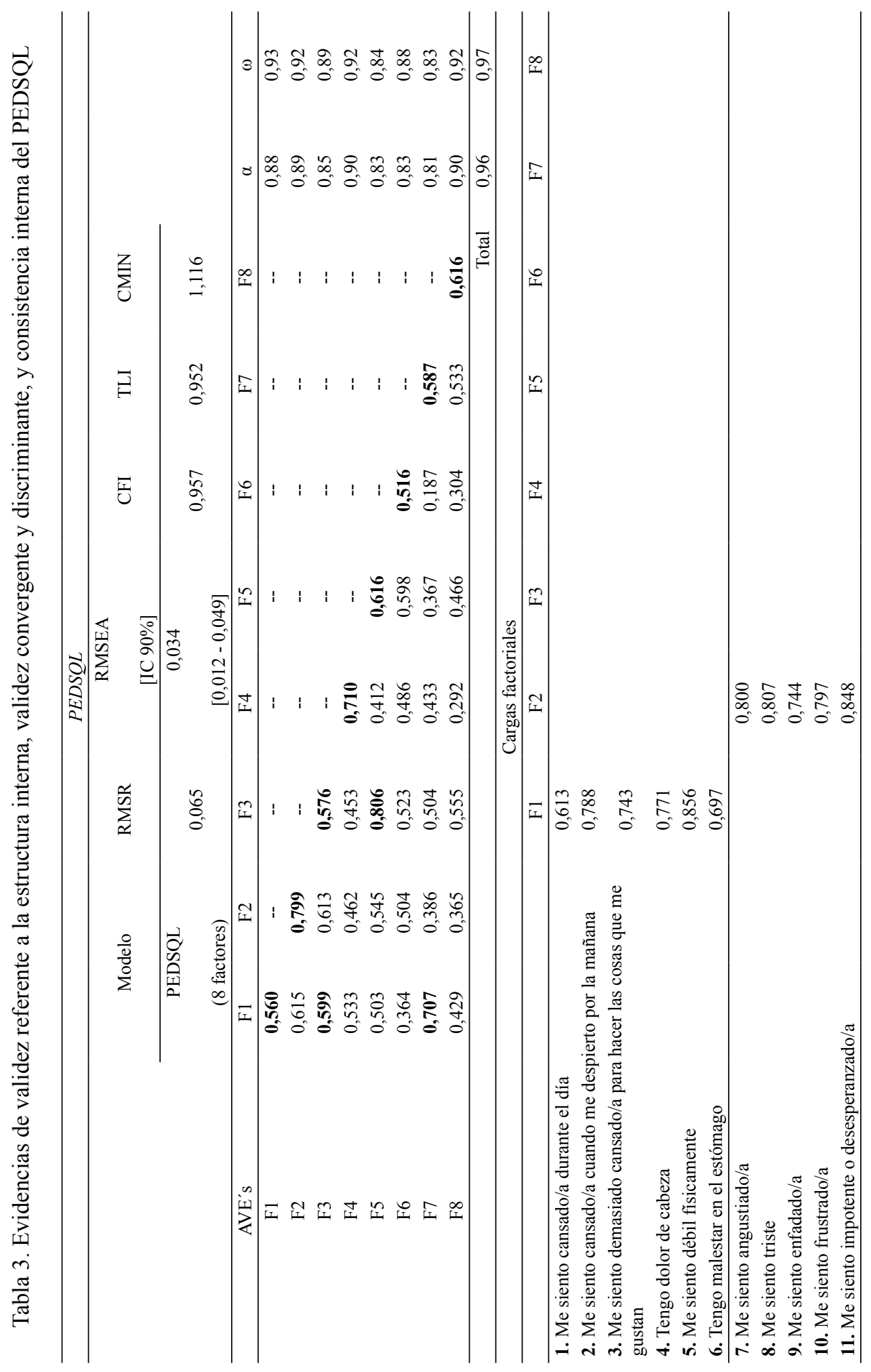




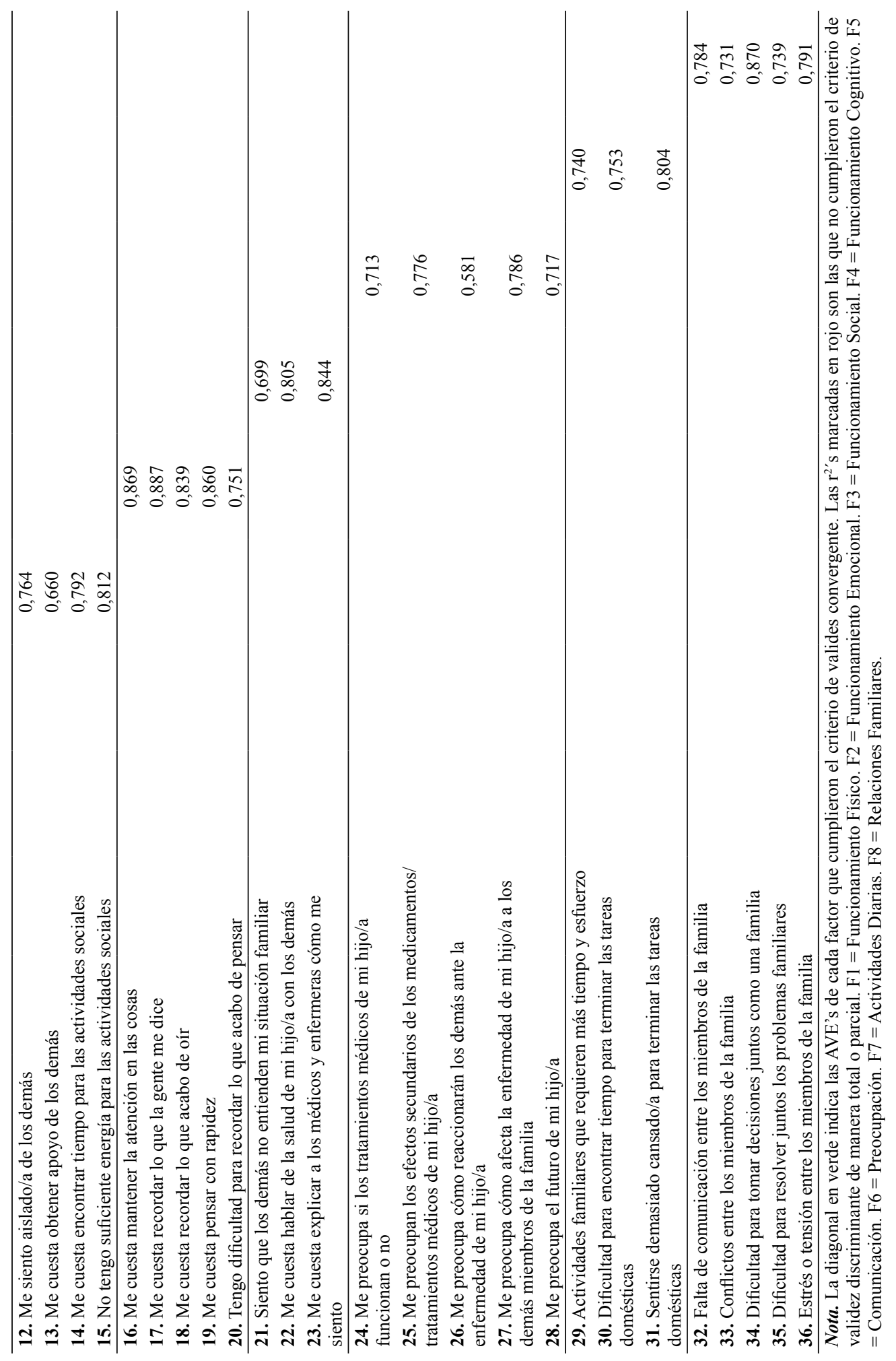


Ambos instrumentos obtuvieron valores de consistencia interna buenos y excelentes, tanto para el coeficiente $\alpha$ como para el $\omega$. El coeficiente $\alpha$ se presenta como un recurso que permite la comparación con otros estudios, no obstante, está bien documentado que su uso no es adecuado en ausencia de ítems paralelos o TauEquivalentes. Por ello, como alternativa robusta para la estimación de consistencia interna se toma el coeficiente $\omega^{(31)}$.

\section{Procedimiento}

Para realizar la presente investigación, se diseñó de manera previa un protocolo de investigación aprobado por los Comités de Investigación y Ética del Instituto Nacional de Pediatría (INP) con el número de registro 2019/039. Para la aplicación de los instrumentos antes descritos se abordaba a los CPIs en el área de hospitalización de oncología y hematología del Instituto Nacional de Pediatría de la Ciudad de México, la aplicación comenzó en abril del 2019 finalizando en julio del mismo año. Para evaluar la claridad y comprensión de las pruebas se realizó un estudio piloto con 10 cuidadores, hecho esto se realizaron modificaciones menores y se prosiguió con la aplicación de manera completa.

La recolección de datos se realizaba de la siguiente manera: la psicóloga acudía a la cama del paciente y se presentaba, reconociendo a aquellos cuidadores que cumplían con los criterios de inclusión antes mencionados, los CPIs eran identificados por medio de la revisión de expedientes, además de referencias por parte de profesionales de salud del área. Se les exponía brevemente el propósito de la investigación y se les invitaba a participar. Posteriormente, a aquellos CPIs que aceptaban colaborar se les presentaba y explicaba la carta de consentimiento informado que contenía las características del estudio, garantía de anonimato, confidencialidad y la libre opción de participar o no en el estudio sin consecuencias institucionales de ninguna índole, se les aclaraban dudas y se les pedía que lo firmarán. Posteriormente, ya firmado el consentimiento informado, a aquellos CPIs que les fuera complicado dejar la habitación debido a la corta edad del infante se les realizaba la entrevista ahí mismo para después entregar la batería de instrumentos, al transcurrir una hora se regresaba a aclarar dudas y se recogían las pruebas, verificando que todos los ítems hubieran sido contestados.

Los cuidadores con pacientes de mayor edad acompañaban a la investigadora a un espacio silencioso y con suficiente luz, en ese espacio se iniciaba la entrevista para posteriormente aplicar las pruebas psicológicas en el siguiente orden: Cuestionario de datos sociodemográficos, Encuesta de Necesidades de Apoyo Insatisfechas de la Persona de Apoyo versión corta (SPUNS) y PedsQL ${ }^{\mathrm{TM}} 2.0$ Módulo de Impacto familiar. La psicóloga se mantenía cerca y pendiente del participante durante toda la evaluación.

La evaluación se realizaba de manera individual y en una sola sesión de aproximadamente 30 minutos. Si el cuidador no sabía leer, se le leían y explicaban los ítems de forma que no quedaban excluidos de la evaluación. Es importante mencionar que el Comité de Ética en Investigación del hospital sugirió el desarrollo de una intervención dirigida a la población de estudio. Atendiendo a esta propuesta, se decidió realizar el diseño e implementación de un Taller Psicoeducativo basado en la evaluación de necesidades de apoyo de los CPIs de pacientes pediátricos con cáncer, en octubre del 2019. 


\section{Análisis estadístico}

Se utilizó el programa estadístico SPSS versión 23 para el análisis estadístico de los datos. Primero, se hizo un análisis descriptivo presentando frecuencias y porcentajes para las variables categóricas, así como media aritmética, máximos, mínimos y desviación estándar para las variables continuas. Se analizó la correlación entre las variables utilizando el coeficiente Rho de Spearman dada la falta de normalidad estadística que presentaron las variables intervalares en la prueba de KolmogorovSmirnov. Se buscaron correlaciones con valores $\mathrm{p} \leq, 05$. Finalmente, se realizó un análisis de regresión múltiple, así como un análisis de Potencia Estadística (PE) por medio del software G*Power versión 3.1.9.3.

\section{Resultados}

\section{Análisis descriptivos}

El tipo de tratamiento para la mayoría de los pacientes era quimioterapia (72\%), y en segundo lugar el tratamiento combinado de quimioterapia, radioterapia y cirugía $(27 \%)$. El $76 \%$ de los pacientes tenía algún hermano y el 22\% había presentado recaídas o recurrencia de la enfermedad. La percepción que tenían los cuidadores en relación con la salud del paciente era en su mayoría buena (63\%), siguiendo de una percepción de salud regular $(21 \%)$, muy buena $(11 \%)$, excelente $(4 \%)$ y pobre $(1 \%)$. Por otro lado, en el último año, únicamente el $10 \%$ de los cuidadores había recibido apoyo psicológico y otro $20 \%$ asesoramiento por parte de una enfermera o trabajadora social.

\section{Características del cuidado en el paciente infantil}

Se encontró que durante la hospitalización los CPIs invierten en el cuidado del paciente un promedio de 10 horas diarias. Cabe resaltar, que el tiempo de cuidado brindado por el cuidador no podía ser compartido con otro familiar debido a las políticas y espacio del hospital. Se reportó que el tiempo transcurrido desde el diagnóstico de la enfermedad hasta el momento del estudio fue de 1 mes hasta 12 años. Véase la Tabla 4.

Tabla 4. Características en el cuidado del paciente infantil $(\mathrm{N}=100)$.

\begin{tabular}{lc}
\hline \multicolumn{1}{c}{ Características } & Frecuencia \\
\hline Tiempo invertido en el cuidado por día & \\
$<=6$ horas & 19 \\
$>6$ horas & 81 \\
Tiempo desde el diagnóstico & 63 \\
$<=1$ año & 32 \\
$1-5$ años & 5 \\
$>5$ años & \\
\hline
\end{tabular}




\section{Necesidades de apoyo insatisfechas}

Con relación a la variable de necesidades de apoyo en el CPI se encontró que las principales necesidades insatisfechas fueron: la necesidad de resolver las preocupaciones por el futuro $(\overline{\mathrm{x}}=59,87)$, seguido por las necesidades emocionales $(\overline{\mathrm{x}}=49,89)$, y las necesidades de información $(\overline{\mathrm{x}}=49,50)$. Para los cuidadores, el factor con mayor grado de satisfacción y menor urgencia por ser resuelto fue la necesidad de acceso médico $(\overline{\mathrm{x}}=40,58)$. Véase la Tabla 5 .

Tabla 5. Medias y desviaciones estándar de subescalas del instrumento de Necesidades de Apoyo Insatisfechas.

\begin{tabular}{lc}
\hline \multicolumn{1}{c}{ Subescalas } & Media \pm SD \\
\hline Necesidades de información & $47,80 \pm 27,70$ \\
Preocupaciones sobre el futuro & $64,33 \pm 31,40$ \\
Necesidades económicas y del trabajo & $56,87 \pm 32,63$ \\
Necesidad de acceso médico & $41,35 \pm 28,22$ \\
Necesidades personales & $44,87 \pm 27,08$ \\
Necesidades emocionales & $53,93 \pm 30,74$ \\
\hline
\end{tabular}

Nota. Altos valores indican mayores necesidades insatisfechas.

Los ítems con menor grado de satisfacción fueron: "Cómo enfrentar la preocupación de que el cáncer empeore" $(\overline{\mathrm{x}}=65,75)$, seguido por "Cómo enfrentar la preocupación de no saber lo que sucederá en el futuro" $(\overline{\mathrm{x}}=64,25)$, "Cómo encontrarle sentido a la experiencia que estoy viviendo con mi hijo/a" $(\overline{\mathrm{x}}=57,5)$, "Cómo luchar con la idea de que mi hijo/a se deje vencer" ( $\overline{\mathrm{x}}=56)$, y "Cómo encontrar información sobre los efectos secundarios de los tratamientos contra el cáncer de mi hijo/a” $(\overline{\mathrm{x}}=54,75)$.

\section{Calidad de vida relacionado con la salud y Repercusiones familiares}

La media del puntaje total del índice de calidad de vida relacionado con la salud fue de 58,72/100. Se encontró que, de los cuatro dominios pertenecientes a la calidad de vida, el funcionamiento emocional $(51,40 / 100)$ fue el más deteriorado, seguido por el área física $(58,66 / 100)$ y social $(60 / 100)$. El puntaje total de impacto familiar fue de 57,59/100, situándose las relaciones familiares como uno de los dominios menos afectados (64/100). El dominio de preocupación obtuvo el puntaje más bajo $(44,55 / 100)$, siendo así, el rubro más deteriorado en todo el instrumento. Véase la Tabla 6. 
Tabla 6. Medias y desviaciones estándar de Puntajes Totales y subescalas del instrumento Módulo de Impacto Familiar.

\begin{tabular}{lcc}
\hline \multicolumn{1}{c}{ Puntajes totales y Subescalas } & Número de ítems & Media \pm SD \\
\hline Total Impacto Familiar & 36 & $57,59 \pm 20,60$ \\
Total Calidad de Vida & 20 & $58,72 \pm 21,31$ \\
Funcionamiento Físico & 6 & $58,66 \pm 24,09$ \\
Funcionamiento Emocional & 5 & $51,40 \pm 25,28$ \\
Funcionamiento Social & 4 & $60,06 \pm 26,12$ \\
Funcionamiento Cognitivo & 5 & $65,05 \pm 24,00$ \\
Comunicación & 3 & $59,33 \pm 29,01$ \\
Preocupación & 5 & $44,55 \pm 24,37$ \\
Total Repercusiones Familiares & 8 & $62,25 \pm 24,96$ \\
Actividades diarias & 3 & $59,33 \pm 27,55$ \\
Relaciones familiares & 5 & $64 \pm 27,54$ \\
\hline
\end{tabular}

Nota. Altos valores indican mejor calidad de vida y funcionamiento familiar.

Los ítems más afectados en el dominio de calidad de vida fueron: "Me siento cansado durante el día" ( $\overline{\mathrm{x}}=45,25)$, "Me siento angustiado" $(\overline{\mathrm{x}}=46)$, y "Me siento triste" $(\overline{\mathrm{x}}=46,25)$. Por otro lado, contemplando el instrumento en su totalidad, los ítems mayormente afectados correspondieron al dominio de preocupación, los cuales fueron: "Me preocupa el futuro de mi hijo/a" $(\overline{\mathrm{x}}=26,25)$ y "Me preocupan los efectos secundarios de los medicamentos" $(\overline{\mathrm{x}}=35)$.

\section{Análisis de relación}

Entre las cuatro dimensiones de necesidades de apoyo con el total de calidad de vida y el total de impacto familiar se encontraron relaciones negativas y con probabilidades menores a 0.01 y 0.05 de obtener correlaciones iguales o más extremas que las obtenidos en este trabajo, en muestras aleatorias de la misma población. El dominio de NA que obtuvo mayor fuerza de asociación con CV fue necesidades emocionales $\left(r_{s}=, 706 ; p=2,4584 \times 10^{-16}\right)$, seguido por necesidades sobre el futuro $\left(r_{s}=, 483 ; p\right.$ $\left.=3,6651 \times 10^{-7}\right)$, necesidades de información $\left(r=, 457 ; p=2 \times 10^{-6}\right)$, y necesidades de acceso de la atención médica $\left(r=, 351 ; p=3,42 \times 10^{-4}\right)$. Otras variables correlacionadas fueron comunicación $\left(r_{s}=, 749 ; p=3,1554 \times 10^{-19}\right)$, preocupación $\left(r_{s}=, 658 ; p=\right.$ $\left.1,0627 \times 10^{-13}\right)$, actividades diarias $\left(r_{s}=, 708 ; p=1,6984 \times 10^{-16}\right)$, relaciones familiares $\left(r_{s}=, 643 ; p=5,4681 \times 10^{-13}\right)$, tiempo brindado al paciente $\left(r_{s}=, 292 ; p=, 003\right), \mathrm{y}$ empleo $\left(r_{s}=, 209 ; p=, 037\right)$.

No obstante, los resultados no arrojaron correlaciones con valores $\mathrm{p} \leq .05$ entre las variables NA y CV con la variable tiempo transcurrido desde el diagnóstico. 
Factores que predicen la calidad de vida del cuidador primario informal

Se realizó un análisis de regresión lineal múltiple con el método de introducción. Se evaluaron y cumplieron los supuestos de 1) no colinealidad ( $F I V=1,75$; Tolerancia $=2,978) ; 2)$ normalidad de residuos $(K-S=, 060, p=, 200) ; 3)$ linealidad entre necesidades emocionales y CV $\left(r_{\text {AjuLineal }}^{2}=485, F=94,255, p=5,1962 \times 10^{-16} \mathrm{vs}\right.$ $r^{2}{ }_{\text {AjuCuadrática }}=, 480, F=46,669, p=6.3312 \times 10^{-15} v s r^{2}{ }_{\text {AjuCúbica }}=, 475, F=30,901, p=$ $\left.4,551 \times 10^{-14}\right)$, linealidad entre necesidades de información y $\mathrm{CV}\left(r_{\text {AjuLineal }}^{2}=, 196, F=\right.$ 25,065, $p=2 \times 10^{-6} v s r_{\text {AjuCuadrática }}^{2}=, 200, F=13,392, p=7 x 10^{-6} v s r^{2}{ }_{\text {AjuCübica }}=, 192$, $\left.F=8,848, p=3,1 \times 10^{-5}\right)$, linealidad entre actividades diarias y $\mathrm{CV}\left(r^{2}{ }_{\text {AjuLineal }}=, 485\right.$, $F=94,050, p=5,4772 \times 10^{-16} v s r_{\text {AjuCuadrática }}^{2}=480, F=46,641, p=6,4229 \times 10^{-15} v \mathrm{~s}$ $\left.r_{\text {AjuCúbica }}^{2}=481, F=31,623, p=2,6465 \times 10^{-14}\right)$, linealidad entre relaciones familiares y CV $\left(r_{\text {AjuLineal }}^{2}=, 408 F=69,264, p=5,1771 \times 10^{-13} v s r_{\text {AjuCuadrática }}^{2}=412, F=35,707\right.$, $p=2,3943 \times 10^{-12}$ vs $\left.r_{A j u C i b i c a}^{2}=410, F=23,896, p=1,2324 \times 10^{-11}\right)$, linealidad entre preocupaciones y $\mathrm{CV}\left(r_{\text {AjuLineal }}^{2}=, 445 F=80,515, p=2,0467 \times 10^{-14} v s r_{\text {AjuCuadrática }}^{2}=\right.$ ,444, $F=40,467, p=1,6633 \times 10^{-13}$ vs $r_{\text {AjuCibica }}^{2}=$,450, $F=28,047, p=4,1264 \times 10^{-}$ ${ }^{13}$ ); 4) homocedasticidad (sin patrón observable en el diagrama de dispersión de PredichosXResiduos); e 5) Independencia de residuos

$(\mathrm{D}-\mathrm{W}=1,980)$.

El modelo mostró que las necesidades emocionales $\left(b_{Z}=-, 341, p=1,97 \times 10^{-4}\right)$, las necesidades de información no satisfechas $\left(b_{z}=, 160, p=, 033\right)$, las actividades diarias $\left(b_{z}=, 399, p=5,6443 \times 10^{-8}\right)$, las relaciones familiares $\left(b_{z}=, 133, p=, 059\right)$ y las preocupaciones de los CPI $\left(b_{Z}=, 334, p=4 \times 10^{-6}\right)$ explican el $74 \%$ de la varianza total de la calidad de vida. El modelo con los cinco predictores obtuvo una probabilidad menor a 1/10,000 de ser más extremo que en este estudio, en muestras aleatorias de la misma población $\left(F_{(5,94)}=58,030, p=3,0806 \times 10^{-27}\right)$. Véase la Tabla 7 .

Tabla 7 . Factores que predicen la calidad de vida del CPI.

$$
\mathrm{R}^{2}=0,742, \mathrm{~F}_{(5,94)}=58,030, \mathrm{p}<0,001
$$

\begin{tabular}{lccccc}
\hline Variables predictoras & $\beta$ & $E E$ & $\beta z$ & $t$ & $P$ \\
\hline Necesidades emocionales &,- 272 &, 070 &,- 341 & $-3,875$ &, 000 \\
Necesidades de información &, 119 &, 055 &, 160 & 2,165 &, 033 \\
Actividades diarias &, 308 &, 052 &, 399 & 5,902 &, 000 \\
Relaciones familiares &, 103 &, 054 &, 133 & 1,911 &, 059 \\
Preocupaciones &, 292 &, 060 &, 334 & 4,900 &, 000 \\
\hline
\end{tabular}

Nota: Análisis de Regresión Múltiple. $\mathrm{EE}=$ Error Estándar. $\beta \mathrm{z}=$ Coeficientes beta estandarizados. Nivel de significación: $\mathrm{p}<0,01$.

\section{Tamaño del efecto y Poder estadístico}

Dado que las correlaciones presentaron en su mayoría un tamaño del efecto o en su equivalente una fuerza de asociación ${ }^{(32,33)}$ de moderada a fuerte, sabemos que 
la magnitud del efecto, asociación y varianza compartida entre las variables fue relevante $^{(34)}$. La potencia estadística encontrada en las correlaciones con fuerza de asociación de moderada a fuerte fueron de 0,99 o más, reflejando una alta potencia estadística en los datos obtenidos ${ }^{(35)}$.

\section{Discusión y conclusiones}

Uno de los objetivos principales del presente estudio fue describir las necesidades de apoyo y calidad de vida del CPI de pacientes pediátricos con cáncer hospitalizados. Esto con motivo de analizar las necesidades de apoyo prioritarias para esta población y brindar diversas recomendaciones para futuras evaluaciones e intervenciones.

Los resultados mostraron la existencia de una variedad de necesidades de apoyo insatisfechas por parte de los cuidadores primarios informales de niños con cáncer. Las preocupaciones por el futuro, seguido por las necesidades emocionales y de información fueron percibidas con una mayor insatisfacción. Por otro lado, las necesidades de acceso de la atención médica fueron valoradas como las más satisfechas y por lo tanto las menos necesarias para resolver. Esto último concuerda con otros estudios, donde se ha observado que los cuidadores se encuentran satisfechos con los servicios y atención médica que recibe el paciente. Sin embargo, como en el presente estudio, a pesar de manifestar esta necesidad como resuelta los CPIs presentan una baja calidad de $\operatorname{vida}^{(36,37)}$.

La calidad de vida de los CPIs de pacientes pediátricos con cáncer se encontró afectada, en particular las áreas de funcionamiento emocional, físico y social. Esto confirma los hallazgos de otros autores en la misma población ${ }^{(36,38,39)}$.

Es importante destacar que, a pesar de que el funcionamiento emocional fuera el rubro más deteriorado, los cuidadores no consideraron que el aprender a manejar su estrés y poder hablar abiertamente de sus preocupaciones con el personal de salud fueran de las necesidades más relevantes. Para poder comprender estos resultados podríamos remontarnos a que la mayoría de los participantes fueron madres de los pacientes, quienes desde un constructo social ya cumplen el papel de cuidadoras en la familia y dada la vulnerabilidad del paciente con cáncer, sus funciones y obligaciones en el rol maternal se intensifican ${ }^{(40)}$. Lo anterior da pie a la deslegitimación de sus propias necesidades y a un escaso nivel de autocuidado emocional y físico que podría explicar la baja percepción de gravedad de sus síntomas, así como una pobre necesidad en obtener apoyo psicológico. Estos resultados se han observado en otros estudios de cuidadores de pacientes adultos con cáncer $^{(41-43)}$, e incluso con CPIs de pacientes con demencia ${ }^{(44)}$.

En el presente estudio las preocupaciones por el futuro fueron el factor de necesidades mayormente insatisfecho, estos hallazgos confirman lo encontrado por autores que evaluaron a cuidadores de pacientes pediátricos con cáncer ${ }^{(10,43,16)}$, los cuales también manifestaron una fuerte necesidad de expresar y manejar el miedo a las posibles recaídas, segundos tumores o muerte de su hijo. En este sentido, se confirma que la incertidumbre está presente desde el comienzo del diagnóstico y esta se encuentra asociada tanto a la evolución del padecimiento como a la respuesta que tenga el paciente ante al tratamiento. De igual manera, aunque la enfermedad y muerte afecte a todos los seres humanos, esta es percibida de modo diferente según la etapa vital en la que se encuentre el individuo. Como padre o madre, la pérdida de 
un hijo implica asumir la muerte de aquellos a quienes tendrían que ayudar a crecer y que habitualmente estarían destinados a sobrevivirles ${ }^{(45)}$.

Por otra parte, los ítems de necesidades emocionales: "Cómo encontrar sentido a la experiencia que estoy viviendo con mi hijo" y "Cómo luchar con la sensación de que mi hijo se venga abajo", fueron percibidas dentro de las necesidades primordiales. De igual manera, como se reportó en el estudio de González ${ }^{(46)}$, la necesidad de encontrar información sobre los efectos secundarios de los tratamientos contra el cáncer infantil fue de las mayormente insatisfechas.

Se observó que la población de este estudio presentó menores puntajes y por lo tanto mayores afectaciones en calidad de vida e impacto familiar, en comparación con estudios que utilizaron el mismo instrumento de medición con padres y madres de niños de población estadounidense ${ }^{(47)}$ y cuidadores de pacientes pediátricos con enfermedades crónicas ${ }^{(48,49)}$. Lo anterior manifiesta una clara afectación, particularmente, en la calidad de vida de los CPIs de niños con cáncer.

Los hallazgos del presente estudio mostraron una alta asociación entre las necesidades de apoyo no satisfechas y una menor calidad de vida en el cuidador. Asimismo, se observó que el tiempo brindado al paciente juega un papel importante en la calidad de vida del cuidador. Un alto porcentaje de los participantes manifestó encontrarse desempleado debido a la cantidad de horas que demanda atender a su paciente, así como el deseo de compartir este tiempo con él. Estos cambios en el financiamiento familiar causados por la enfermedad también fueron reportados en otros estudios $(2,11,14,50)$.

Por otro lado, se encontró que la variable de calidad de vida se ve influenciada por las necesidades emocionales y de información, además de las actividades diarias, las relaciones familiares y las preocupaciones de los CPIs. Estas últimas dos coinciden con los recursos de afrontamiento del Modelo del CPIs de población infantil de Raina et al. ${ }^{(51)}$, refiriendo que el buen manejo del estrés y óptimas relaciones familiares pueden beneficiar la salud física y emocional del cuidador y por lo tanto su CV. Por otro lado, la comunicación efectiva y empática por parte del profesional médico es vital en el proceso de cuidado, se ha observado que uno de los principales estilos de afrontamiento en los CPIs es la búsqueda de información tendiendo a ser mayor que en cuidadores de niños con diabetes o epilepsia ${ }^{(52)}$. Además, apoyarles con alternativas para cubrir sus necesidades de tiempo para sus propias actividades beneficiara su experiencia de cuidado.

Por el contrario, el tiempo transcurrido desde el diagnóstico no mostró correlaciones ni diferencias clínicamente importantes ni estadísticamente sugerentes con las variables del estudio, esto pudo deberse a que más de la mitad de los participantes evaluados tenían un año o menos de haber sido diagnosticados.

Vale la pena resaltar la necesidad de que las intervenciones se ajusten a las condiciones socioeconómicas y culturales de los participantes. En este sentido, los recursos materiales que se utilizan deben elaborarse en términos del lenguaje y comprensión de la población a la que va dirigido.

Será importante, para estudios futuros, realizar la evaluación con una mayor cantidad de participantes, con el fin de obtener datos más generalizables. Además, el estudio fue de tipo transversal, lo que no permitió observar cambios en los resultados de las variables a lo largo del tiempo. Continúa siendo necesario la realización de estudios longitudinales con grupos de comparación que permitan determinar en qué medida el desarrollo del funcionamiento psicológico y familiar se ve influido por el 
cáncer infantil, incluyendo la oportunidad de observar los cambios de interacción entre los cuidadores e infantes ${ }^{(19)}$.

Por otra parte, en la presente investigación sólo fue posible acercarse a CPIs de pacientes hospitalizados, sería conveniente evaluar a cuidadores de pacientes ambulatorios en estudios posteriores. Otro aspecto a destacar es que, debido a la remodelación del hospital, durante los meses de aplicación de instrumentos se cambió de piso a toda la sección de oncología ocasionando cierta incomodidad en algunas cuidadoras. Lo anterior puede representar una limitación del estudio, ya que pudo alterar los resultados en las pruebas, así como el acceso de la psicóloga con todos los CPIs disponibles.

Con base en los resultados encontrados, se recomienda diseñar intervenciones que involucren de manera preferente aspectos como: el manejo de la incertidumbre, estrategias de regulación emocional, así como psicoeducación referente a la importancia del autocuidado y los beneficios del apoyo emocional. Igualmente, es imprescindible informar a los CPIs acerca de las características, fases y modalidades de tratamiento del cáncer infantil.

Es evidente que los hallazgos del estudio demostraron una alta prevalencia de necesidades de apoyo no satisfechas que impactan en la CV de los cuidadores de población infantil con cáncer. Por lo tanto, el atender estas necesidades en sus diferentes factores permitirá que su $\mathrm{CV}$ se vea beneficiada. El estudio sustenta la importancia de integrar la detección de necesidades de los CPIs a la atención clínica de rutina en todas las fases de tratamiento. Finalmente, es imprecindible el apoyo psicológico por parte de profesionales de salud mental, así como las intervenciones psicoeducativas periódicas, no sólo para el paciente pediátrico con cáncer sino también para su principal cuidador.

\section{Referencias bibliográficas}

1. Crespo C, Santos S, Tavares A, Salvador Á. Care that matters: Family-centered care, caregiving burden, and adaptation in parents of children with cancer. Fam Syst Health 2016;34: 31-40. doi:10.1037/fsh0000166

2. Rubira E, Marcon S, Belasco A, Gaiva M, Espinosa M. Burden and quality of life of caregivers of children and adolescents with chemotherapy treatment for cancer. Acta Paul Enferm 2012; 25: 567-73.

3. Al-Gamal E, Long T, Shehadeh J. Health satisfaction and family impact of parents of children with cancer: a descriptive cross-sectional study. Scand J Caring Sc 2019;33:81523.

4. Rocha RS, Pinheiro LP, Oriá MO, Ximenes LB, Pinheiro AK, Aquino PS. Social determinants of health and quality of life of caregivers of children with cancer. Determinantes sociais da saúde e qualidade de vida de cuidadores de crianças com câncer. Rev Gaucha Enferm 2016;37:e57954. doi:10.1590/1983-1447.2016.03.57954

5. Kudubes AA, Bektas M, Ugur O. Symptom frequency of children with cancer and parent quality of life in Turkey. Asian Pac J Cancer Prev 2014;15:3487-93. doi:10.7314/ apjcp.2014.15.8.3487

6. WHO. World Health Organization Ageing and Health Programme \& Milbank Memorial Fund. Hacia un consenso internacional sobre la política de atención de salud a largo 
plazo en el envejecimiento. 2000. [Internet]. [Acceso 09 Sep 2018]. Disponible en https:// apps.who.int/iris/handle/10665/66542

7. De los Reyes M. Construyendo el concepto de cuidador de ancianos. [Internet]. 2001 [Acceso 10 Ago 2019]. Disponible en https://gerincon.files.wordpress.com/2010/05/ construyendo_el_concepto_de_cuidador.pdf

8. Montero X, Jurado S, Méndez J. Variables que predicen la aparición de sobrecarga en cuidadores primarios informales de niños con cáncer. Psicooncología 2015; 12:67-86. doi: 10.5209/rev_PSIC.2015.v12.n1.48905

9. Duran B. Developing a scale to measure parental worry and their attitudes toward childhood cancer after successful completion of treatment: a pilot study. J Pediatr Oncol. Nurs 2011; 28:154-168. doi:10.1177/1043454210397755.

10. Llantá M, Hernández K, Martínez Y. Calidad de Vida encuidadores primarios de pacientes oncopediátricos. Instituto Nacional de Oncología y Radiobiología. Rev Haban Cienc Méds 2015; 14:97-106.

11. Kim Y, Kashy D, Spillers R, Evans T. Needs assessment of family caregivers of cancer survivors: three cohorts comparison. Psychooncology 2010; 19:573-582. Doi: 10.1002/ pon. 1597

12. Hileman J, Lackey N, Hassanein R. Identifying the needs of home caregivers of patients with cancer. Oncol Nurs Forum 1992;19, 771-7.

13. Hudson P, Trauer T, Graham S, Grande G, Ewing G, Payne S, Stajduhar K, Thomas K. A systematic review of instruments related to family caregivers of palliative care patients. Palliat Med 2010; 24:656-668. doi: 10.1177/0269216310373167

14. Sloper P. Needs and responses of parents following the diagnosis of childhood cancer. Child: Care Health Dev 1996; 22:187-202.

15. Shields G, Schondel, C, Barnhart L, Fitzpatrick V, Sidell N, Adams P, Fertig B, Gomez S. Social work in pediatric oncology: a family needs assessment. Soc Work in Health Care 1995; 21:39-54. doi: 10.1300/J010v21n01_04

16. Yiu J, Twinn S. Determining the needs of Chinese parents during the hospitalization of their child diagnosed with cancer: An exploratory study. Cancer Nurs 2001; 24: 483-9. nDoi: 10.1097/00002820-200112000-00011

17. Kruger S. Parents in crisis: helping them cope with a seriously ill child. $J$ Pediatr Health Care 1992; 7: 133-140.

18. Tarr J, Pickler R. Becoming a cancer patient: a study of families of children with acute lymphocytic leukemia. J. Pediat Oncol Nurs 1999;16:44-50.

19. Pai A, Greenley R, Lewandowski A, Drotar D, Youngstrom E, Peterson C. A metaanalytic review of the influence of pediatric cancer on parent and family functioning. $\mathrm{J}$ Fam Psychol 2007; 21:407. doi: 10.1037/0893-3200.21.3.407

20. De la Huerta R, Corona J, Méndez J. Evaluación de los estilos de afrontamiento en cuidadores primarios de niños con cáncer. Rev Neurol Neurocir Psiquiat 2006; 39:46-51.

21. Douvoba S, Aguirre R, Infante C, Martínez I, Pérez R. Needs of caregivers of cancer patients: validation of the Mexican version of the Support Person Unmet Needs Survey (SPUNS-SFM). Support Care Cancer 2015; 23: 2925-35. doi: 10.1007/s00520-0152658-0

22. Campbell S, Carey M, Sanson-Fisher R, Barker D, Turner D, Taylor-Brown J, Hall A. Measuring the unmet supportive care needs of cancer support persons: the development of the Support Person's Unmet Needs Survey - short form. Eur J Cancer Care 2014; 23:255-262. doi: 10.1111/ecc. 12138 
23. Varni W, Sherman S, Burwinkle M, Dickinson P, Dixon P. The PedsQL ${ }^{\mathrm{TM}}$ Family Impact Module: Preliminary reliability and validity. Health Qual Life Outcomes 2004; 2:55 doi:10.1186/1477-7525-2-55

24. Mapi Research Institute. Linguistic validation of the PedsQLTM- a Quality of Life Questionnaire Research and Evaluation, Limited Use translation of PedsQL ${ }^{\mathrm{TM}}$. [Internet]. [Acceso 26 Abr 2018]. Disponible en https://www.pedsql.org/PedsQL-LinguisticValidation-Guidelines.doc

25. WHOQOL. World Health Organization Quality of Life Assessment: position paper from the World Health Organization. Soc Sci Med 1995; 41:1403-9.

26. Abad F, Olea J, Ponsoda V, García C. Medición en ciencias sociales y de la salud. Editorial Síntesis; 2011.

27. Brown, T. Confirmatory factor analysis for applied research. Guilford Press. 2015.

28. Cheung G, Wang C. Current approaches for assessing convergent and discriminant validity with SEM: issues and solutions. Academy of Management Proceedings 2017; 12706. doi: 10.5465/AMBPP.2017.12706abstract

29. Fornell C, Larcker D. Evaluating structural equation models with unobservable variables and measurement error. Journal of Marketing Research 1981; 18: 39-50. doi: $10.2307 / 3151312$

30. George D, Mallery P. IBM SPSS Statistics 23 step by step. A simple guide and reference. Allyn and Bacon, 2017. doi: 10.4324/9781315545899

31. Dunn T, Baguley T, Brunsden V. From alpha to omega: A practical solution to the pervasive problem of internal consistency estimation. Br J Psychol 2013; 105: 399-412. doi:10.1111/bjop. 12046

32. Kline, R. Beyond significance testing. Washington, DC: American Psychological Association; 2004.

33. Vacha-Haase T, Thompson B. How to estimate and interpret various effect sizes. J Couns. Psychol 2004; 51:473-481.

34. Ferguson C. An effect size primer: A guide for clinicians and researchers. Prof Psychol Res Pr 2009; 40:532-8. doi:10.1037/a0015808

35. Pérez-Tejada, H. Estadística para las ciencias sociales, del comportamiento y de la salud. México: CENGAGE Learning; 2008.

36. Al-Gamal E, Long T, Shehadeh J. Health satisfaction and family impact of parents of children with cancer: a descriptive cross-sectional study. Scand J Caring Sc 2019;33:81523.

37. Ullrich A, Ascherfeld L, Marx G, Bokemeyer C, Bergelt C, Oechsle K. Quality of life, psychological burden, needs, and satisfaction during specialized inpatient palliative care in family caregivers of advanced cancer patients. BMC Palliat Care 2017;16:31.

38. Rubira E, Marcon S, Belasco A, Gaiva M, Espinosa M. Burden and quality of life of caregivers of children and adolescents with chemotherapy treatment for cancer. Acta Paul Enferm 2012; 25: 567-73.

39. Santo E, Gaíva M, Espinosa M, Barbosa D,Belasco A. Cuidando del niño con cáncer: evaluación de la sobrecarga y calidad de vida de los cuidadores. Rev Lat Am de Enfermagem 2011; 19, 3.

40. Young B, Dixon-Woods M, Findlay M, Heney D. Parenting in a crisis: conceptualising mothers of children with cancer. Soc Sci Med 2002; 55:1835-47.

41. Dionne-Odom J, Applebaum A, Ornstein K, Azuero A, Warren P, Taylor R, Rocque G, Kvale E, Demark-Waahnefried W, Pisu M, Partridge E, Martin M, Bakitas M. Participation 
and interest in support services among family caregivers of older adults with cancer. Psychooncology 2017; 27:969-76. doi: 10.1002/pon.4603

42. Merckaert I, Libert Y, Lieutenant F, Moucheaux A, Farvacques C, Slachmuylder J, Razavi D. Desire for formal psychological support among caregivers of patients with cancer: prevalence and implications for screening their needs. Psychooncology 2012; 22:138995. doi: 10.1002/pon.315

43. Shields G, Schondel, C, Barnhart L, Fitzpatrick V, Sidell N, Adams P, Fertig B, Gomez S. Social Work in Pediatric Oncology: A Family Needs Assessment. Soc Work in Health Care 1995; 21:39-54. doi: 10.1300/J010v21n01_04

44. Rosa E, Lussignoli G, Sabbatini F, Chiappa Ā, Di Cesare S, Lamanna L, Zanetti O. Needs of caregivers of the patients with dementia. Arch Gerontol Geriat 2010; 51:54-8. doi:10.1016/j.archger.2009.07.008

45. Barreto P. Soler M. Muerte y Duelo. Madrid, España: Síntesis; 2007.

46. González P. Experiencias y necesidades percibidas por los niños y adolescentes con cáncer y por sus familias. Nure Inv 2005;16.

47. Medrano G, Berlin K, Davies, W. Utility of the PedsQL ${ }^{\text {TM }}$ family impact module: assessing the psychometric properties in a community sample. Qual. Life Res 2013; 22:2899-2907. doi: 10.1007/s11136-013-0422-9

48. Panepinto J, Hoffmann R, Pajewski N. A psychometric evaluation of the PedsQL ${ }^{\mathrm{TM}}$ Family Impact Module in parents of children with sickle cell disease. Health Qual life outcomes 209; 7(1):32.

49. Hurtado J, Álvarez G. Calidad de vida relacionada con la salud del niño y del adolescente con obesidad. Salud mental 2014; 37: 119-25.

50. Pereira MS, Magni RH, Mingione IC, Caran EM. Os desafios diários do cuidador de crianças e de adolescentes com câncer. Prat Hosp (São Paulo) 2009; 11:51-3.

51. Raina P, O’Donnell M, Schwellnus H, Rosenbaum P, King G, Brehaut, J, Russell D, Swinton M, King S, Wong M, Walter, S, Wood E. Caregiving process and caregiver burden: conceptual models to guide research and practice. BMC Pediatrics 2004; 4(1), 1.

52. Goldbeck L. Parental coping with the diagnosis of childhood cancer: gender effects, dissimilarity within couples, and quality of life. Psychooncology 2001; 10:325-35. doi: $10.1002 /$ pon 